\title{
Current Search: Performance Evaluation and Application to DC Motor Speed Control System Design
}

\author{
Deacha Puangdownreong \\ Department of Electrical Engineering, South-East Asia University, Bangkok, Thailand \\ Email: deachap@sau.ac.th
}

Received September 27, 2012; revised October 27, 2012; accepted November 4, 2012

\begin{abstract}
This paper proposes the current search (CS) metaheuristics conceptualized from the electric current flowing through electric networks for optimization problems with continuous design variables. The CS algorithm possesses two powerful strategies, exploration and exploitation, for searching the global optimum. Based on the stochastic process, the derivatives of the objective function is unnecessary for the proposed CS. To evaluate its performance, the CS is tested against several unconstrained optimization problems. The results obtained are compared to those obtained by the popular search techniques, i.e., the genetic algorithm (GA), the particle swarm optimization (PSO), and the adaptive tabu search (ATS). As results, the CS outperforms other algorithms and provides superior results. The CS is also applied to a constrained design of the optimum PID controller for the dc motor speed control system. From experimental results, the CS has been successfully applied to the speed control of the dc motor.
\end{abstract}

Keywords: Current Search; Metaheuristics; PID Controller; DC Motor Speed Control System

\section{Introduction}

Over the last five decades, many metaheuristic algorithms have been developed to solve combinatorial and numeric optimization problems. They have been also successfully applied to solve various real-world engineering optimization problems. Most metaheuristic algorithms are based on the stochastic process. This means that the derivatives of the objective function is unnecessary. Unlike the conventional or classical algorithms, they are usually based on the deterministic process commonly using the derivatives (or gradient information) to find the optimum solution. By literatures, metaheuristics can be classified in many ways depending on their nature, for example, evolutionary; nature-inspired, bio-inspired, and physic-inspired; population-based and single-solution (trajectory-) based $[1,2]$. The most powerful metaheuristics should have at least two major properties, i.e., exploration (or diversification) and exploitation (or intensification). Exploration or diversification means to generate diverse solutions to explore the search space on the global scale. Exploitation or intensification means to focus on the search in a local region by exploiting the information to reach the best local solution within this region [2,3]. Population-based metaheuristics algorithms, such as genetic algorithm (GA) [4,5], ant colony optimization (ACO) [6], particle swarm optimization (PSO) [7], harmony search (HS) [8], firefly search (FS) [9], hunting search (HuS) [10], cuckoo search (CuS) [11,12], and bat-inspired search
(BS) [13], have strong explorative property, while single-solution based metaheuristics algorithms, such as simulated annealing (SA) $[14,15]$, tabu search (TS) $[16,17]$, and adaptive tabu search (ATS) $[18,19]$, have strong exploitation characteristics.

In 2012, the current search (CS) metaheuristics was proposed to solve optimization problems [20]. The CS algorithms are conceptualized from the electric current flowing through electric networks. It has been successfully applied to design the PID controllers for unstable systems [21], and the PIDA controllers for hard-to-becontrolled systems [22]. This paper consists of five sections. The CS algorithms are explained in senses of exploration and exploitation properties as appeared in Section 2. The CS metaheuristics is conducted to evaluate its performance via testing against several well-known unconstrained optimization problems. Results obtained are compared among the genetic algorithm (GA), the particle swarm optimization (PSO), and the adaptive tabu search (ATS) as presented in Section 3. The CS is applied to a constrained design of the optimum PID controller for the dc motor speed control. The experimental setup, results, and discussions are illustrated in Section 4, while conclusions follow in Section 5.

\section{Current Search Algorithms}

Algorithms of the CS metaheuristics are conceptualized from the electric current flowing through electric net- 
works. The behavior of electric current is like a tide that always flow from higher to lower places. The less the resistance of blanch in electric networks, the more the current flows. All blanches connected in networks represent the feasible solutions in search space. The local entrapment is occurred when the current hits the open network connection. The optimum solution found is located at the end of the optimum current path. Algorithms of the CS are described as follows.

Step 1. Initialize the search space $\Omega$, iteration counter $k=j=l=1$, maximum allowance of solution cycling $j_{\max }$, number of initial solutions (feasible current path in a network) $N$, number of neighborhood members $n$, maximum objective function value $\varepsilon$, search radius $R$, and set $\Psi=\Gamma$ $=\Xi=\varnothing$.

Step 2. Uniformly random initial solution $\boldsymbol{X}_{i}, i=1, \cdots$, $N$ within $\Omega$. Evaluate the objective function $f\left(\boldsymbol{X}_{i}\right)$ of $\forall \boldsymbol{X}$ Rank $\boldsymbol{X}_{i}, i=1, \cdots, N$ giving $f\left(X_{1}\right)<\cdots<f\left(X_{N}\right)$, then store ranked $\boldsymbol{X}_{i}$ into set $\Psi$.

Step 3. Let $\boldsymbol{x}_{0}=\boldsymbol{X}_{k}$ as selected initial solution.

Step 4. Uniformly random neighborhood $x_{k, l}, l=1, \cdots$, $n$ around $x_{0}$ within radius $R$. Evaluate the objective function $f\left(x_{k, l}\right)$ of $\forall \boldsymbol{x}$. A solution giving the minimum objective function is set as $x^{*}$.

Step 5. If $f\left(x^{*}\right)<f\left(x_{0}\right)$, keep $x_{0}$ in set $\Gamma_{k}$ and set $x_{0}=x^{*}$, set $j=1$ and return to Step 4. Otherwise keep $x^{*}$ in set $\Gamma_{k}$ and update $j=j+1$.

Step 6. If $f\left(x_{0}\right)<\varepsilon$, adjust radius $R=\rho R, \rho \in[0,1]$ to speed up the search process.

Step 7. If $j<j_{\max }$, return to Step 4. Otherwise keep $\boldsymbol{x}_{0}$ in set $\Xi$ and update $k=k+1$.

Step 8. Stop the search process when termination criteria (TC) are satisfied. The optimum solution found is $\boldsymbol{x}_{0}$ Otherwise return to Step 3.

The CS algorithms can be summarized by the pseudo code as shown in Figure 1 and can be represented by the flow diagram in Figure 2. In Step 1, the search space $\Omega$ performs the feasible boundary where the electric current can flow. The maximum allowance of solution cycling $j_{\text {max }}$ implies the local entrapment occurred in the selected path. The number of initial solutions $N$ is set as feasible paths of the electric currents. The number of neighborhood members $n$ provides the sub-directions of the electric currents in the selected path, and the search radius $R$ is given as the sub-search space where the electric current can flow in the selected path. $\Psi, \Gamma$, and $\Xi$ are provided as the memory lists to store ranked initial solutions, solutions found in each search path, and the best solution in each search path, respectively.

The uniformly random approach is conducted in Step 2 to perform the feasible path of the electric currents within $\Omega$. These paths will be ranked by the objective function to arrange the signification of paths from most to

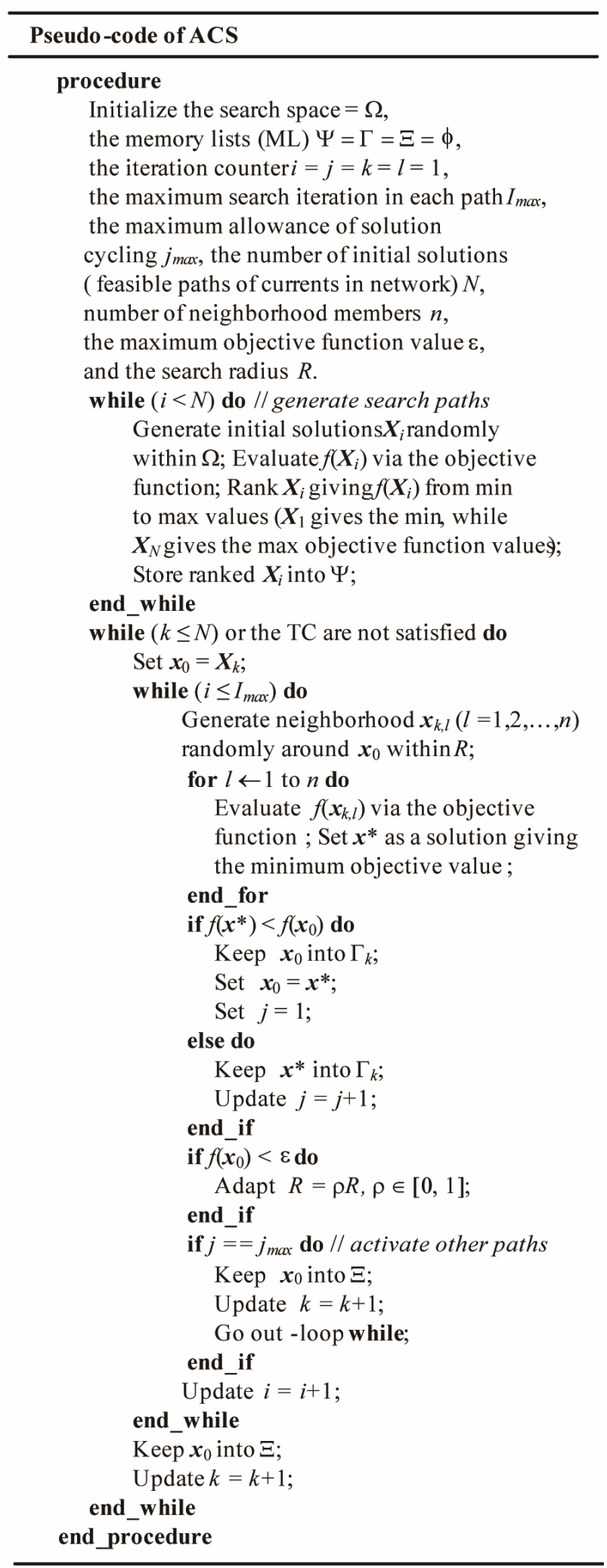

Figure 1. Pseudo code of the CS algorithms.

least. This operation is regarded as the exploration property to generate diverse solutions to explore the search 


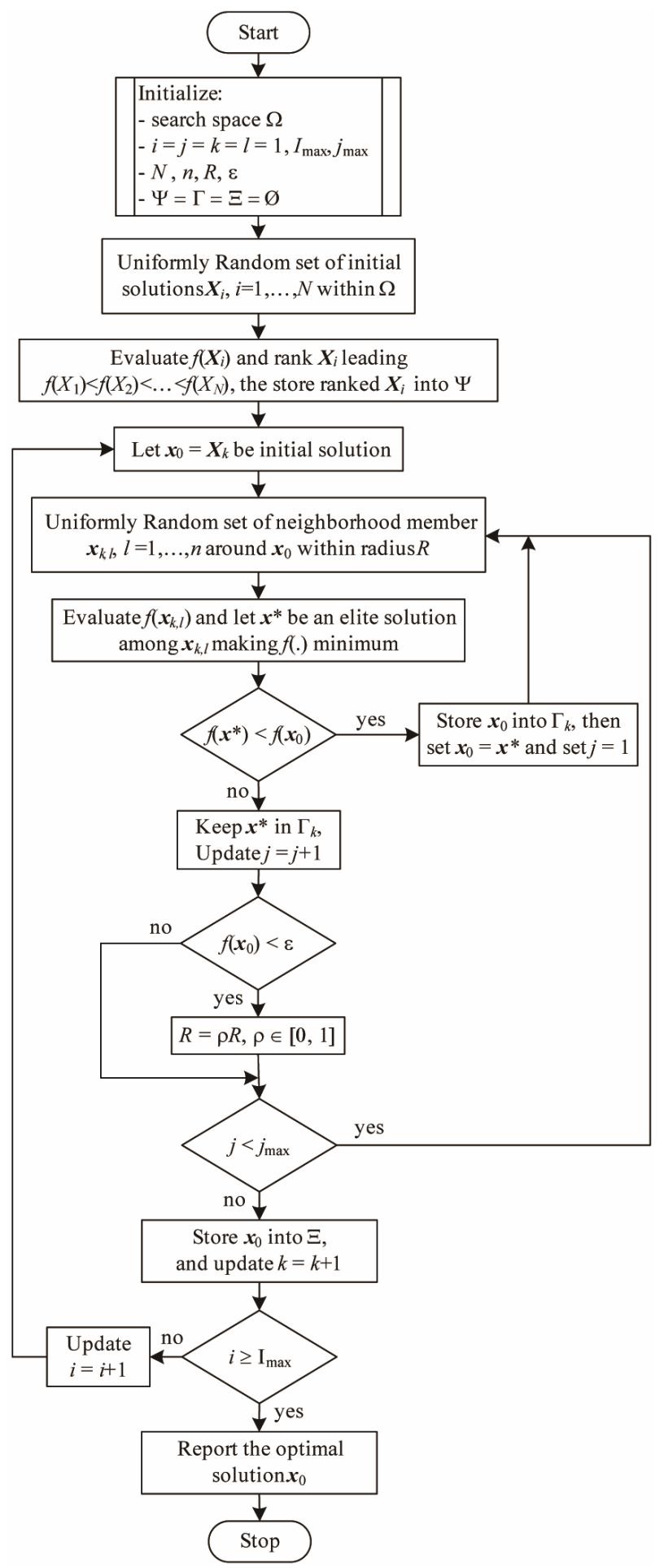

Figure 2. The flow diagram of the CS algorithms.

space on the global scale. The more the numbers of initial solutions, the more the diverse solutions are generated over the search space. Once the most significant path of the current is selected in Step 3 - 5, the search process will consecutively find the optimum solution within the sub-search space.
In Step 6, the search radius $R$ is gradually decreased when the search comes close to local or global solutions. In this situation, the shorter the search radius, the greater the probability of the optimum solution can be found. This operation is regarded as the exploitation property focusing on the search by exploiting the information to reach the best local solution within a local region.

In Step 7 - 8, each feasible solution will be evaluated via the objective function until the optimum solution is found. The local entrapment in the selected path will be identified via the maximum allowance of solution cycling. If occurred, the second, the third, and so on, of the significant paths ranked in Step 2 will consecutively employed, until optimum solution will be found or the TC will be met.

Generally, the TC can be defined in many ways depending on the particular problems. One TC can be determined as the maximum number of search iterations which can be defined from the ratio of search space to search radius. There are other TC approaches, such as maximum number of objective function evaluation, maximum iteration without improvement in solution quality, and maximum CPU time, for example. In engineering optimization problems, TC usually used is the sufficient solution quality concept represented by the cost $J$. The value of the cost $J$ depends on applications and can be set from design specifications, component tolerances, and so on.

According to CS algorithms, exploration and exploitation properties can complement each other and make the CS most powerful. Algorithms of the CS are elaborately described for the readers to follow and general enough for applications to various optimization problems.

\section{Performance Evaluation}

This section presents the performance comparison studies among genetic algorithm (GA), particle swarm optimization (PSO), adaptive tabu search (ATS), and current search (CS). Algorithms of the GA, the PSO, and the ATS are omitted. Readers may refer to good sources $[4,5]$ for the GA, [7] for the PSO, and [18,19] for the ATS, respectively. GA, PSO, ATS, and CS are tested against eight well-known unconstrained optimization problems [23] including Bohachevsky function (BF), the fifth function of De Jong (DJF), Griewank function (GF), Michaelwicz function (MF), Rastrigin function (RF), Salomon function (SalF), Schwefel function $(\mathrm{SchF})$, and Shekel's fox-holes function (SF). Table 1 summarizes these test functions in which $J_{\text {min }}$ is the minimum values of objective functions required to terminate the search.

Algorithms of PSO, ATS, and CS were coded by MATLAB running on Intel Core2 Duo $2.0 \mathrm{GHz} 3$ Gbytes DDR-RAM computer, while GA is used from the MAT- 
Table 1. Summary of the unconstrained optimization problems.

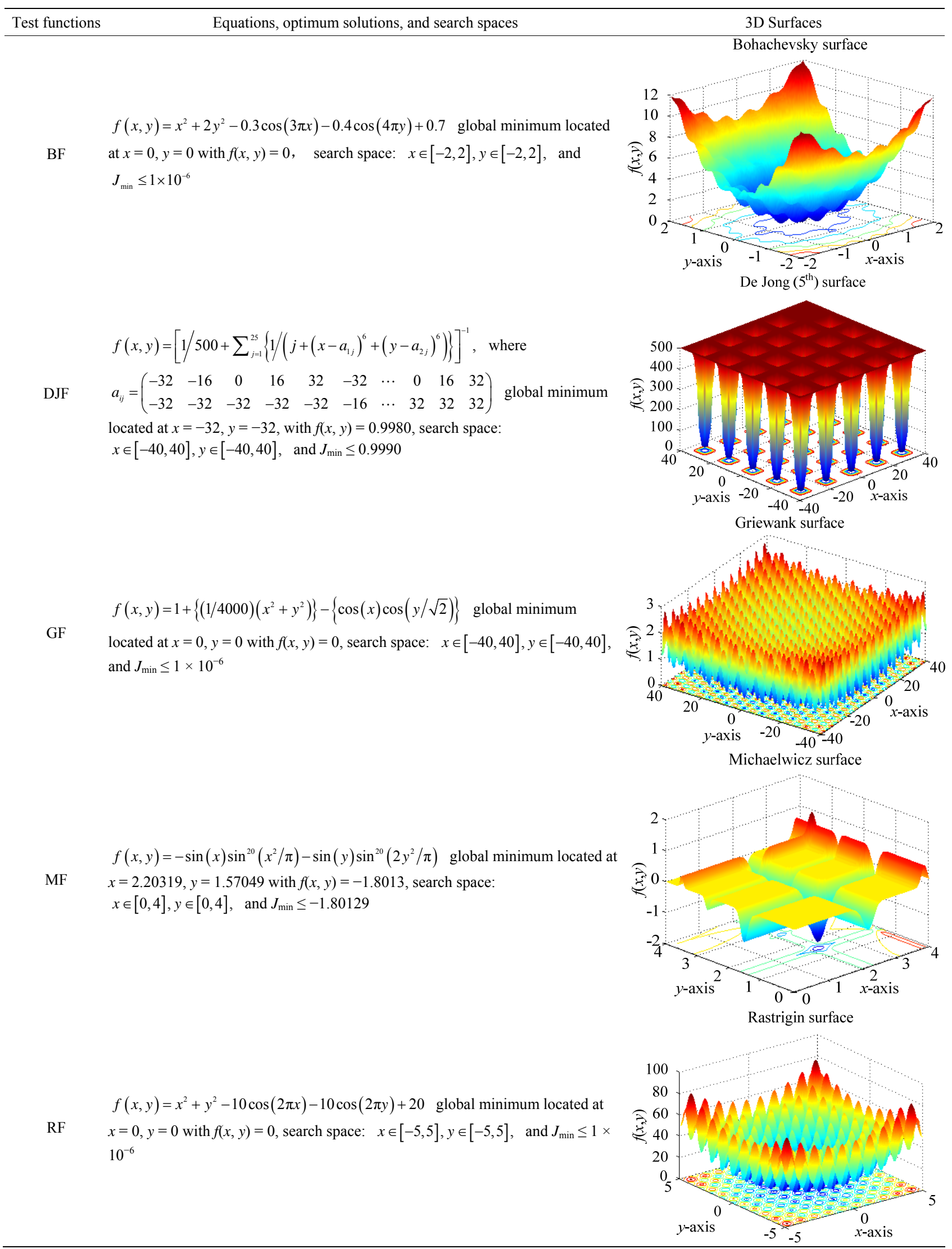




\section{Continued}

SalF

$$
f(x, y)=1-\cos \left(2 \pi \sqrt{x^{2}+y^{2}}\right)+0.1 \sqrt{x^{2}+y^{2}} \text { global minimum located at } x=0,
$$

$y=0$ with $f(x, y)=0$, search space: $x \in[-4,4], y \in[-4,4]$, and $J_{\min } \leq 1 \times 10^{-6}$

$f(x, y)=837.9658-\{x \sin (\sqrt{x})+y \sin (\sqrt{y})\}$ global minimum located at

$\operatorname{SchF}$

$x=420.9687, y=420.9687$ with $f(x, y)=0$, search space:

$x \in[-500,500], y \in[-500,500]$, and $J_{\min } \leq 1 \times 10^{-6}$
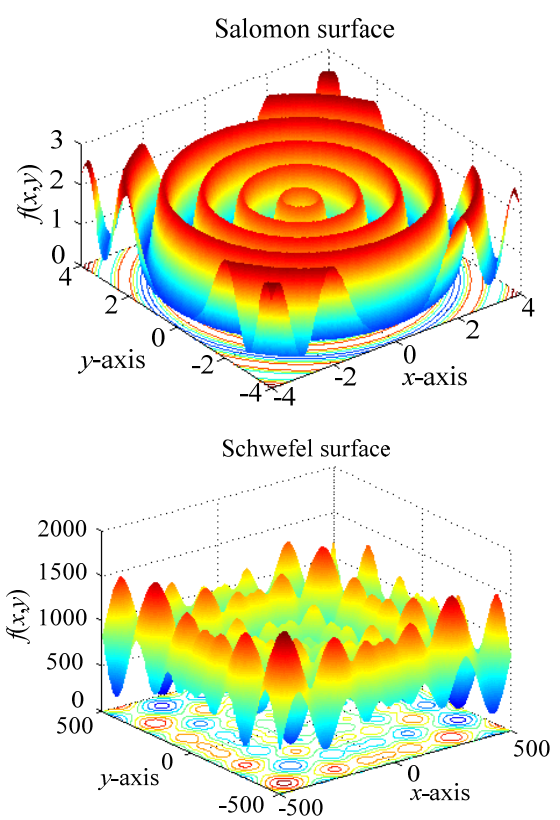

Shekel's fox-holes surface

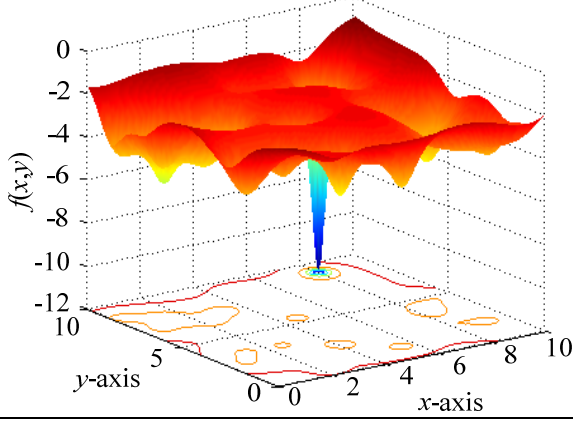

LAB-GA Toolbox [5]. Each of these algorithms performs search on each test function for 50 trials. Each search trial begins the search with different initial solutions, while search parameters are kept the same for all trials. This approach is commonly referred to as multiplepoints-single-strategy (MPSS) in metaheuristic contexts. Search parameter settings for the GA follow MATLABGA Toolbox [5], for the PSO follow [7], for the ATS follow $[18,19]$. Tables 2 and 3 summarize the search parameters of the ATS and the CS respectively.

The averages results over 50 trials are summarized in Table 4. There are two groups of data, i.e., average number of objective function evaluations and average search time. Referring to the search time data in Table 4, the CS spends search time of $58.27 \%$ less than the GA does, $47.79 \%$ less than the ATS does, and $47.72 \%$ less than the PSO does as average. Referring to the number of objective function evaluations in Table 4, the CS evaluates the objective function of $58.26 \%$ less than the GA does, $47.49 \%$ less than the ATS does, and $47.72 \%$ less than the PSO does as, respectively.

Table 5 summarizes the solutions obtained from those algorithms. It can be noticed that the CS outperforms other algorithms and provides solutions with the best quality within the shortest search time. This outstanding performance of the CS is achieved due to its explorative and exploitative characteristics. Figure 3 represents the CS movements over the search spaces of test functions. It was found that the large areas of all search spaces are explored by the explorative characteristic of the CS. In Figure 3, the CS can provide a high-quality solution rapidly because the elite search path can be provided. The search rapidly focuses the search to the solution with its exploitative characteristic.

The CS metaheuristics has been applied to a constrained parametric search problem, that is, design of the optimum PID controller for the dc motor speed control which is widely used in industries. In the next section, experimental setup and implementation results are illustrated. 
Table 2. ATS parameters.

\begin{tabular}{|c|c|c|c|c|c|c|}
\hline \multirow{2}{*}{$\begin{array}{c}\text { Test } \\
\text { functions }\end{array}$} & \multirow{2}{*}{$N$} & \multirow{2}{*}{$R$} & \multirow{2}{*}{$\begin{array}{c}B T \\
\left(n \_r e \_\max \right)\end{array}$} & \multirow{2}{*}{ Count $_{\max }$} & \multicolumn{2}{|c|}{$A R$} \\
\hline & & & & & Stage I & Stage II \\
\hline $\mathrm{BF}$ & 50 & 0.1 & 5 & 10,000 & $J<1 \times 10^{-2} \rightarrow R=0.01$ & $J<1 \times 10^{-4} \rightarrow R=0.001$ \\
\hline DJF & 100 & 5.0 & 5 & 10,000 & $J<1 \times 10^{2} \rightarrow R=1.00$ & $J<10 \rightarrow R=0.50$ \\
\hline GF & 100 & 0.5 & 5 & 10,000 & $J<1 \times 10^{-2} \rightarrow R=0.01$ & $J<1 \times 10^{-4} \rightarrow R=0.001$ \\
\hline MF & 50 & 2.0 & 5 & 10,000 & $J<1 \rightarrow R=0.01$ & $J<0 \rightarrow R=0.001$ \\
\hline $\mathrm{RF}$ & 100 & 0.5 & 5 & 10,000 & $J<1 \times 10^{-3} \rightarrow R=0.001$ & $J<1 \times 10^{-4} \rightarrow R=0.0001$ \\
\hline SalF & 100 & 0.5 & 5 & 10,000 & $J<1 \times 10^{-3} \rightarrow R=0.001$ & $J<1 \times 10^{-4} \rightarrow R=0.0001$ \\
\hline $\operatorname{SchF}$ & 100 & 10.0 & 5 & 10,000 & $J<1 \times 10^{-2} \rightarrow R=0.001$ & $J<1 \times 10^{-4} \rightarrow R=0.0001$ \\
\hline $\mathrm{SF}$ & 100 & 1.5 & 5 & 10,000 & $J<-2 \rightarrow R=1.00$ & $J<-5 \rightarrow R=0.50$ \\
\hline
\end{tabular}

Notes: $N=$ number of neighborhood members, $R=$ search radius, $B T$ (back-tracking), $n \_r e \_$max $=$maximum allowance of solution cycling before invoking $B T$, Count $_{\max }=$ maximum search iterations, $A R$ (adaptive search radius) $[18,19]$.

Table 3. CS parameters.

\begin{tabular}{|c|c|c|c|c|c|c|}
\hline \multirow{2}{*}{$\begin{array}{c}\text { Test } \\
\text { functions }\end{array}$} & \multirow{2}{*}{$n$} & \multirow{2}{*}{$R$} & \multirow{2}{*}{$N$} & \multirow{2}{*}{$I_{\max }$} & \multicolumn{2}{|c|}{$R$-adjustment } \\
\hline & & & & & Stage I & Stage II \\
\hline $\mathrm{BF}$ & 50 & 0.1 & 50 & 1000 & $J<1 \times 10^{-2} \rightarrow R=0.01$ & $J<1 \times 10^{-4} \rightarrow R=0.001$ \\
\hline DJF & 50 & 5.0 & 100 & 1000 & $J<1 \times 10^{2} \rightarrow R=1.00$ & $J<10 \rightarrow R=0.50$ \\
\hline GF & 100 & 0.5 & 100 & 1000 & $J<1 \times 10^{-2} \rightarrow R=0.01$ & $J<1 \times 10^{-4} \rightarrow R=0.001$ \\
\hline MF & 50 & 2.0 & 50 & 1000 & $J<1 \rightarrow R=0.01$ & $J<0 \rightarrow R=0.001$ \\
\hline $\mathrm{RF}$ & 100 & 0.5 & 100 & 1000 & $J<1 \times 10^{-3} \rightarrow R=0.001$ & $J<1 \times 10^{-4} \rightarrow R=0.0001$ \\
\hline SalF & 100 & 0.5 & 200 & 1000 & $J<1 \times 10^{-3} \rightarrow R=0.001$ & $J<1 \times 10^{-4} \rightarrow R=0.0001$ \\
\hline $\operatorname{SchF}$ & 100 & 10.0 & 100 & 1000 & $J<1 \times 10^{-2} \rightarrow R=0.001$ & $J<1 \times 10^{-4} \rightarrow R=0.0001$ \\
\hline SF & 100 & 1.5 & 50 & 1000 & $J<-2 \rightarrow R=1.00$ & $J<-5 \rightarrow R=0.50$ \\
\hline
\end{tabular}

Notes: $n=$ number of neighborhood members, $R=$ search radius, $N=$ search (current) paths, $I_{\max }=$ maximum search iterations.

Table 4. Average results over 50 trials.

\begin{tabular}{ccccccccc}
\hline \multirow{2}{*}{$\begin{array}{c}\text { Test } \\
\text { functions }\end{array}$} & \multicolumn{3}{c}{ Average number of function evaluations } & \multicolumn{3}{c}{ Average search time (seconds) } \\
\cline { 2 - 9 } & GA & PSO & ATS & CS & GA & PSO & ATS & CS \\
\hline BF & 480,100 & 465,100 & 424,123 & 200,300 & 6.52 & 6.32 & 5.76 & 2.72 \\
DJF & 358,400 & 352,520 & 375,485 & 114,222 & 20.50 & 18.75 & 19.67 & 6.07 \\
GF & 902,150 & 780,464 & 752,260 & 501,500 & 11.67 & 10.10 & 9.74 & 6.49 \\
MF & 200,375 & 145,680 & 126,450 & 50,100 & 3.01 & 2.18 & 1.89 & 0.75 \\
RF & 495,560 & 474,725 & 468,526 & 240,150 & 9.80 & 9.39 & 9.27 & 4.75 \\
SalF & 913,250 & 610,500 & 585,375 & 454,100 & 17.46 & 11.67 & 11.19 & 8.68 \\
SchF & 405,345 & 248,654 & 286,140 & 150,250 & 5.69 & 3.49 & 4.12 & 2.11 \\
SF & 685,450 & 545,120 & 601,150 & 320,300 & 12.07 & 9.60 & 10.59 & 5.64 \\
\hline
\end{tabular}


Table 5. Results obtained from GA, PSO, ATS, and CS.

\begin{tabular}{|c|c|c|c|c|c|}
\hline $\begin{array}{c}\text { Test } \\
\text { functions }\end{array}$ & Results & GA & PSO & ATS & CS \\
\hline \multirow{4}{*}{$\mathrm{BF}$} & Min & $2.1250 \times 10^{-8}$ & $1.3651 \times 10^{-9}$ & $1.4563 \times 10^{-9}$ & $1.9790 \times 10^{-10}$ \\
\hline & Max & $3.4613 \times 10^{-7}$ & $6.4197 \times 10^{-8}$ & $4.0219 \times 10^{-8}$ & $1.5602 \times 10^{-9}$ \\
\hline & Average & $1.0312 \times 10^{-7}$ & $5.7490 \times 10^{-9}$ & $6.0498 \times 10^{-9}$ & $3.0564 \times 10^{-10}$ \\
\hline & Std. & $1.0140 \times 10^{-7}$ & $5.6873 \times 10^{-9}$ & $6.1012 \times 10^{-9}$ & $1.1102 \times 10^{-10}$ \\
\hline \multirow{4}{*}{ DJF } & Min & 0.9980 & 0.9980 & 0.9980 & 0.9980 \\
\hline & Max & 0.9989 & 0.9985 & 0.9982 & 0.9981 \\
\hline & Average & 0.9984 & 0.9983 & 0.9981 & 0.9980 \\
\hline & Std. & 0.0003 & 0.0002 & 0.0001 & 0.0001 \\
\hline \multirow{4}{*}{ GF } & Min & $4.0248 \times 10^{-8}$ & $2.4597 \times 10^{-9}$ & $2.8471 \times 10^{-9}$ & $1.0142 \times 10^{-10}$ \\
\hline & Max & $2.0527 \times 10^{-7}$ & $4.6894 \times 10^{-8}$ & $1.8210 \times 10^{-8}$ & $2.4992 \times 10^{-9}$ \\
\hline & Average & $6.0141 \times 10^{-7}$ & $7.0315 \times 10^{-9}$ & $8.4010 \times 10^{-9}$ & $4.6617 \times 10^{-10}$ \\
\hline & Std. & $5.9414 \times 10^{-7}$ & $6.5143 \times 10^{-9}$ & $4.9834 \times 10^{-9}$ & $1.4652 \times 10^{-10}$ \\
\hline \multirow{4}{*}{ MF } & Min & -1.8013 & -1.8013 & -1.8013 & -1.8013 \\
\hline & Max & -1.8012 & -1.8012 & -1.8012 & -1.8012 \\
\hline & Average & -1.8012 & -1.8012 & -1.8013 & -1.8013 \\
\hline & Std. & 0.0002 & 0.0002 & 0.0001 & 0.0001 \\
\hline \multirow{4}{*}{$\mathrm{RF}$} & Min & $3.9830 \times 10^{-8}$ & $4.0539 \times 10^{-9}$ & $4.0143 \times 10^{-9}$ & $1.2322 \times 10^{-10}$ \\
\hline & Max & $4.8527 \times 10^{-7}$ & $3.3127 \times 10^{-8}$ & $1.0381 \times 10^{-8}$ & $1.0103 \times 10^{-9}$ \\
\hline & Average & $1.4291 \times 10^{-7}$ & $6.9973 \times 10^{-9}$ & $6.3967 \times 10^{-9}$ & $3.8730 \times 10^{-10}$ \\
\hline & Std. & $1.6608 \times 10^{-7}$ & $2.9978 \times 10^{-9}$ & $5.9910 \times 10^{-9}$ & $1.9195 \times 10^{-10}$ \\
\hline \multirow{4}{*}{ SalF } & Min & $4.0462 \times 10^{-8}$ & $5.6563 \times 10^{-9}$ & $6.4251 \times 10^{-9}$ & $1.0102 \times 10^{-9}$ \\
\hline & Max & $6.0985 \times 10^{-7}$ & $4.1027 \times 10^{-8}$ & $4.2879 \times 10^{-8}$ & $2.4510 \times 10^{-8}$ \\
\hline & Average & $8.0302 \times 10^{-7}$ & $7.9410 \times 10^{-9}$ & $8.0693 \times 10^{-9}$ & $4.0301 \times 10^{-9}$ \\
\hline & Std. & $7.9878 \times 10^{-7}$ & $6.9901 \times 10^{-9}$ & $7.9902 \times 10^{-9}$ & $4.1207 \times 10^{-9}$ \\
\hline \multirow{4}{*}{ SchF } & Min & $1.1374 \times 10^{-7}$ & $5.1503 \times 10^{-8}$ & $4.0470 \times 10^{-8}$ & $1.8547 \times 10^{-8}$ \\
\hline & Max & $8.1302 \times 10^{-7}$ & $3.4532 \times 10^{-7}$ & $2.0604 \times 10^{-7}$ & $2.0021 \times 10^{-7}$ \\
\hline & Average & $4.4531 \times 10^{-7}$ & $8.0831 \times 10^{-8}$ & $6.8876 \times 10^{-8}$ & $2.5654 \times 10^{-8}$ \\
\hline & Std. & $5.8523 \times 10^{-7}$ & $7.6574 \times 10^{-8}$ & $7.0130 \times 10^{-8}$ & $1.9983 \times 10^{-8}$ \\
\hline \multirow{4}{*}{ SF } & Min & -12.1190 & -12.1190 & -12.1190 & -12.1190 \\
\hline & Max & -12.1189 & -12.1189 & -12.1189 & -12.1189 \\
\hline & Average & -12.1189 & -12.1189 & -12.1189 & -12.1190 \\
\hline & Std. & 0.0002 & 0.0002 & 0.0002 & 0.0001 \\
\hline
\end{tabular}




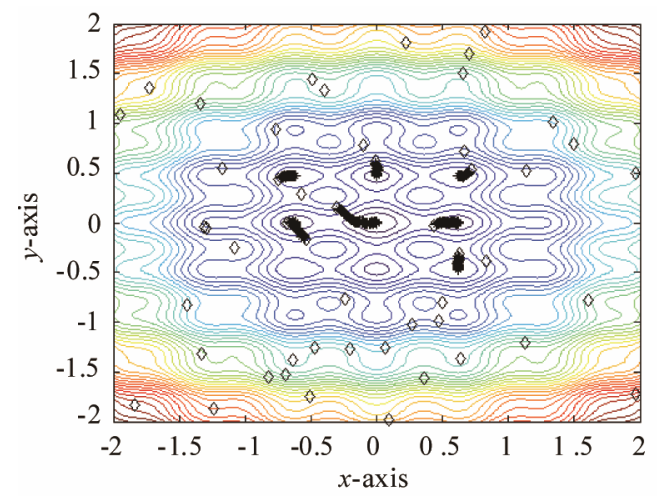

(a)

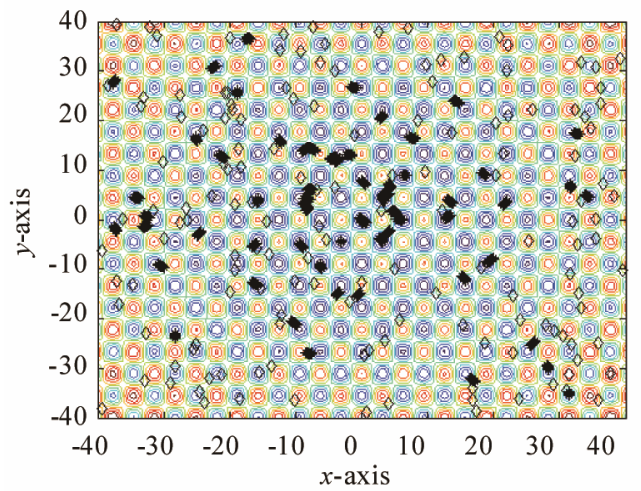

(c)

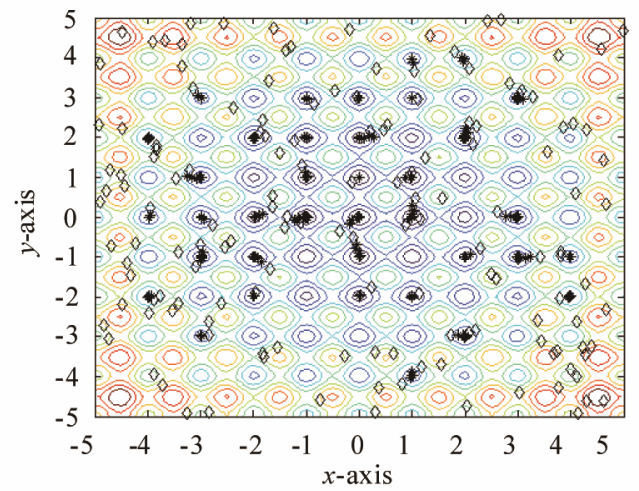

(e)

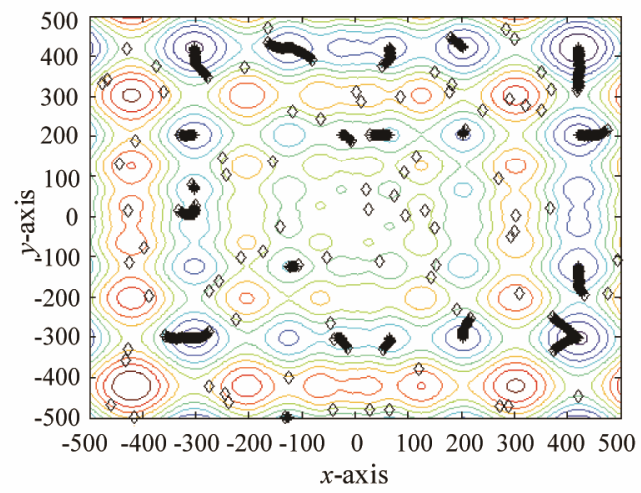

(g)

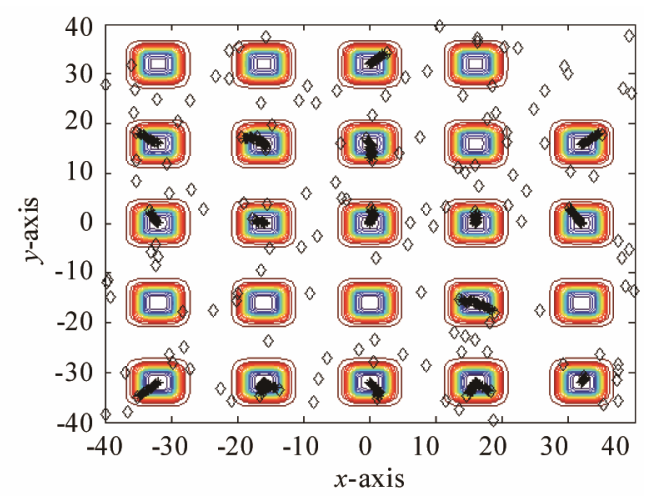

(b)

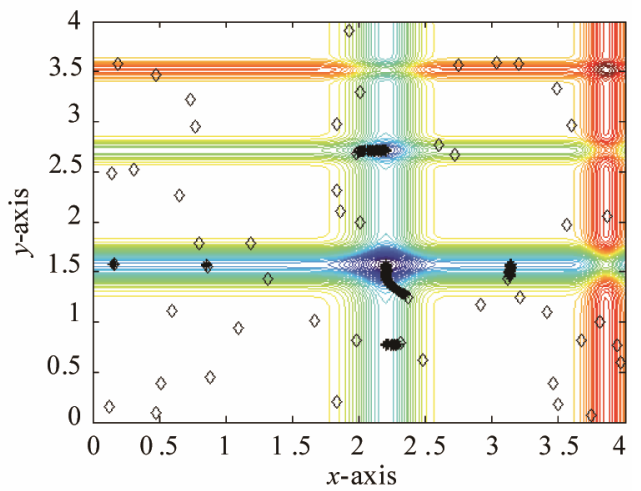

(d)

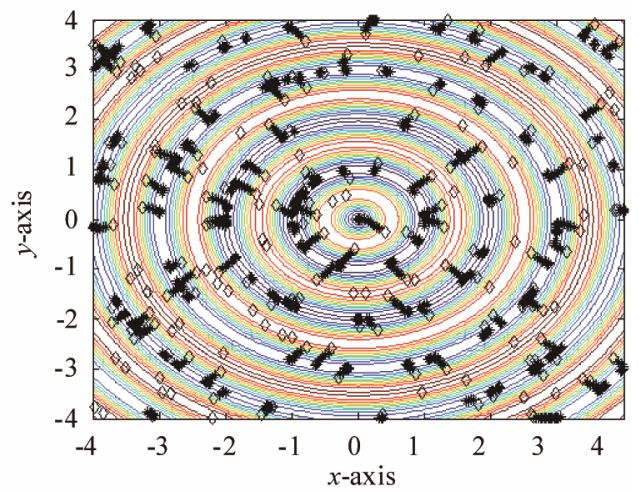

(f)

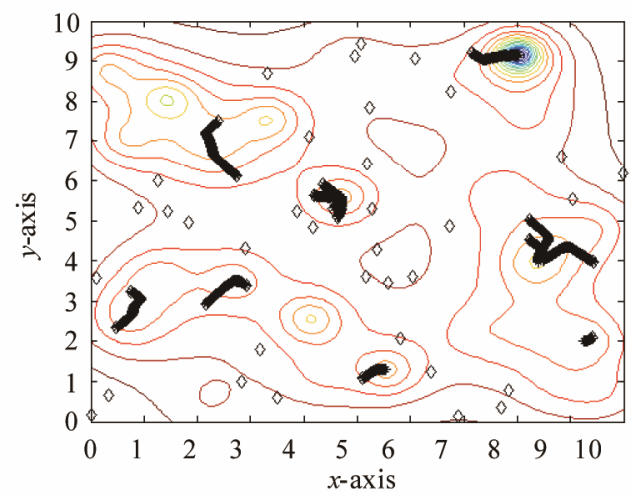

(h)

$\diamond$ representing the explorative solutions

* representing the exploitative solutions

Figure 3. CS movements: (a) BF; (b) DJF; (c) GF; (d) MF; (e) RF; (f) SalF; (g) SchF; and (h) SF. 


\section{DC Motor Speed Control Design}

In this section, the CS is applied to design an optimum PID controller for the dc motor speed control system. This section can be divided into four parts, i.e., mathematical model, model identification, design of PID controller by the CS, and experimental results, respectively. Details are described as follows.

\subsection{Mathematical Model}

The schematic diagram of armature-controlled dc motor can be represented in Figure 4 [24], where $R_{a}$ is an armature-winding resistance, $L_{a}$ is an armature-winding inductance, $R_{f}$ is a field-winding resistance, $L_{f}$ is a fieldwinding inductance, $J$ is a moment of inertia, $B$ is a viscous-friction coefficient, $e_{a}(t)$ is an applied armature voltage, $i_{a}(t)$ is an armature current, $e_{f}(t)$ is a field voltage, $i_{f}(t)$ is a field current, $e_{b}(t)$ is a back emf (electromotive force) voltage, $T(t)$ is a motor torque, $\theta(t)$ is an angular displacement, and $\omega(t)$ is an angular velocity (speed), respectively.

Mathematical model of armature-controlled dc motor in term of the transfer function can be formulated by the differential equations and Laplace transform. The transfer function of armature-controlled dc motor speed control is stated in Equation (1) [24], where $K_{t}$ is a torque constant and $K_{b}$ is a back emf constant.

$$
\frac{\Omega(s)}{E_{a}(s)}=\frac{K_{t}}{J L_{a} s^{2}+\left(B L_{a}+J R_{a}\right) s+B R_{a}+K_{t} K_{b}}
$$

\subsection{Model Identification}

The controlled plant consists of dc motor (LEYBOLDDIDACTIC GMBH, Type 731-91, 0.3 kW, $220 \mathrm{~V}, 2.2 \mathrm{~A}$, $2000 \mathrm{rpm}$ ), an actuator (SCR full-wave controlled rectifier), a speed sensor (tachogenerator LEYBOLD, Type 731-09), and a lowpass filter circuit. Model identification process starts with testing the controlled plant by step input excitation. A step-transient response test was con-

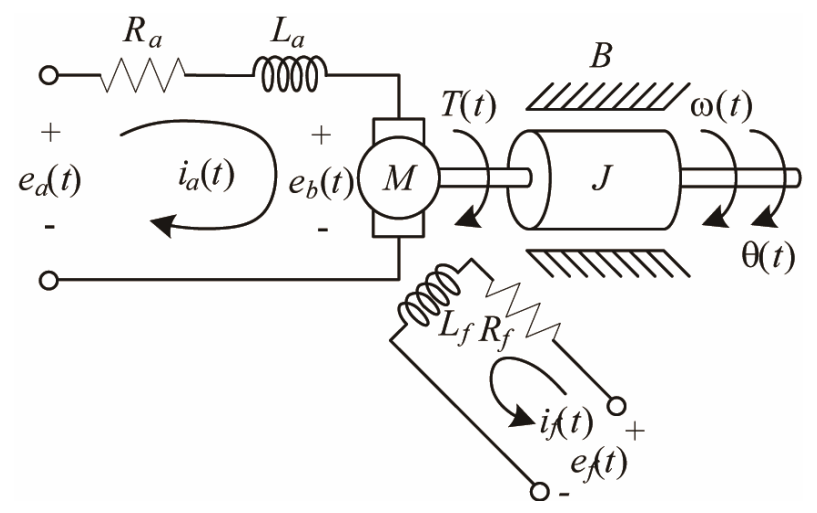

Figure 4. Schematic diagram of dc motor. ducted for the identification of the motor model at the $1860 \mathrm{rpm}$ operating point as shown in Figure 5. It was found that the controlled plant gives the rise time of 2.50 sec, steady-state level of $4 \mathrm{~V}(1860 \mathrm{rpm})$, and without overshoot. The identification using MATLAB and System Identification Toolbox [25] provides the normalized 3rd-order plant model expressed by Equation (2). Figure 6 depicts the simulation step response obtained by the model. The simulation result agrees very well with the experimental one. Therefore, the identified model in Equation (2) is very good representations of the controlled plant.

$$
\left.G_{p}(s)\right|_{\text {plant }}=\frac{1}{1 \times 10^{-5} s^{3}+0.04534 s^{2}+0.5524 s+1}
$$

\subsection{Design of PID Controller by CS}

The CS-based PID controller design for the dc motor speed control system can be performed by the block diagram representation as shown in Figure 7. The theoreticcal function of the PID controller is expressed in Equation (3), where $K_{p}$ is the proportional gain, $K_{i}$, is the integral gain, and $K_{d}$ is the derivative gain, respectively. The

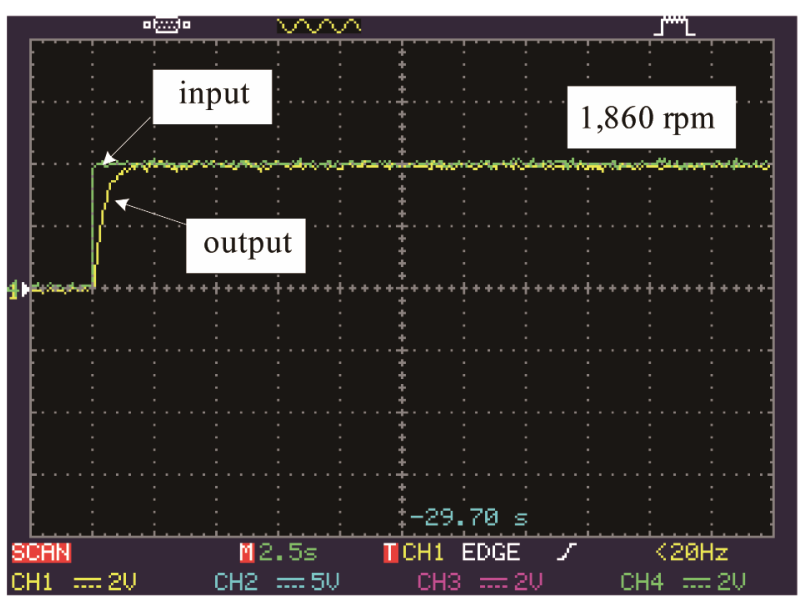

Figure 5. DC motor step response.

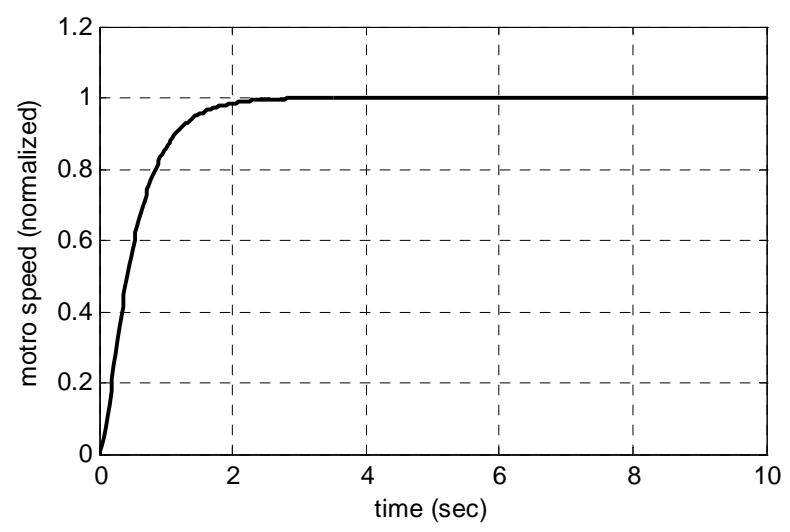

Figure 6. Step response of the plant model. 


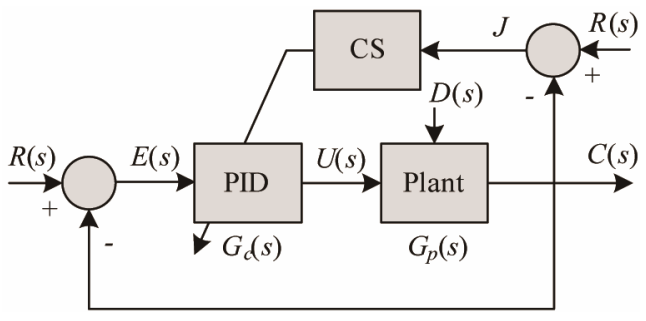

Figure 7. CS-based PID design.

objective function, $J$, the sum of absolute errors between $R(s)$ and $C(s)$ as stated in Equation (4), will be fed back to the CS tuning block. $J$ is minimized to find the optimum PID controller's parameters, i.e., $K_{p}, K_{i}$, and $K_{d}$, giving satisfactory responses.

$$
\begin{gathered}
\left.G_{c}(s)\right|_{\mathrm{PID}}=K_{p}+\frac{K_{i}}{s}+K_{d} s \\
J=\sum_{t=0}^{T}|r(t)-c(t)| \\
\text { min } J=\sum_{t=0}^{T}|r(t)-c(t)| \\
\text { subject to } \quad t_{r} \leq 1.00 \mathrm{sec} . \\
M_{p} \leq 10.00 \%, \\
t_{s} \leq 2.50 \mathrm{sec} ., \\
e_{s s} \leq 1 \times 10^{-5}, \\
0.1 \leq K_{p} \leq 10, \\
0.1 \leq K_{i} \leq 10, \\
0.0001 \leq K_{d} \leq 0.001
\end{gathered}
$$

Referring to Figure 7, the objective function $J$ is minimized according to inequality constraints in Equation (5), where $t_{r}$ is rise time, $M_{p}$ is maximum percent overshoot, $t_{s}$ is settling time, and $e_{s s}$ is steady state error. The CS search parameters are priory set as follows: the maximum search iteration of each path $I_{\max }=200$, the number of initial solutions $N=10$, the number of neighborhood members $n=20$, and the search radius $R=20 \%$ of search spaces. Algorithms of the CS were coded by MATLAB running on Intel Core2 Duo $2.0 \mathrm{GHz} 3$ Gbytes DDR-RAM computer. The tests were conducted 20 trial runs to obtain the optimum PID controller for the dc motor speed control system.

After the search process stopped, the CS successfully provides the optimum PID controller for the motor control system as stated in Equation (6), where $K_{p}=1.6501$, $K_{i}=4.0986$, and $K_{d}=0.0049$, within $216.3504 \mathrm{sec}$ of average search time consumed. The step input and the step disturbance responses of controlled system are depicted in Figure 8. As results, the system without PID controller gives $t_{r}=2.50 \mathrm{sec}$. and cannot be recovered once step disturbance of 0.3 (30\% of unit step) is applied

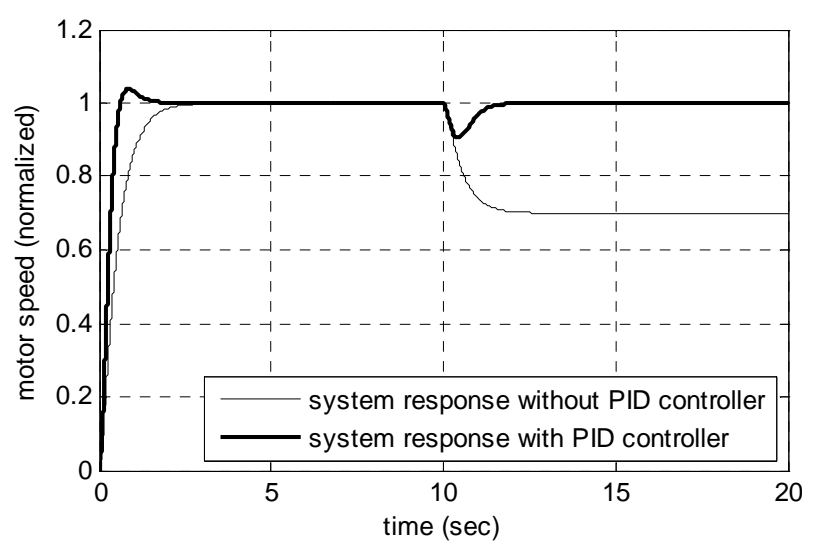

Figure 8. Response of the controlled system.

at the 10th second. This causes the $e_{s s}$ of 0.3 at steadystate response. For the system with PID controller obtained by the CS, the step input response gives $t_{r}=$ $0.8506 \mathrm{sec}, M_{p}=4.04 \%, t_{s}=2.0104 \mathrm{sec}$, and $e_{s s}=0.0$, while the step disturbance response, once the step disturbance of 0.3 is applied, provides the recovering time $t_{r e}=1.8542 \mathrm{sec}, M_{p}=8.24 \%$, and $e_{s s}=0.0$. It was found that the optimum PID controller can be successfully achieved by the CS according to inequality constraints stated in Equation (5).

$$
\left.G_{c}(s)\right|_{\mathrm{PID}}=1.6501+\frac{4.0986}{s}+0.0049 s
$$

\subsection{Experimental Results}

To obtain the experimental results, a closed loop dc motor speed control system with PID controller is a necessary test bed. The diagram in Figure 9 represents the experimental setup. The optimum PID controller obtained by the CS is realized by electronic circuits (op-amp LM335 and RC network) to ensure real-time operation. Driving the motor requires a full-wave controlled rectifier controlled by IC-TCA785. Signal conditioning circuits including lowpass filter and zero-span circuits are also conducted. The experimental setup installed in the laboratory is shown in Figure 10, while the experimental results are depicted in Figure 11.

Referring to Figure 11, it was found that the controlled dc motor speed control system with PID controller designed by the CS can provide the very satisfactory results in both command following and load regulating (disturbance rejection) modes. In command following mode, the system response gives $t_{r}=0.98 \mathrm{sec}, M_{p}=$ $5.01 \%, t_{s}=2.42 \mathrm{sec}$, and $e_{s s}=0.0$, while in disturbance rejection mode once the step disturbance of 0.3 is applied the system provides the recovering time $t_{r e}=1.55 \mathrm{sec}, M_{p}$ $=7.85 \%$, and $e_{s s}=0.0$. The system responses obtained from the test bed agree very well with those obtained from the model simulation. Very good agreement be- 


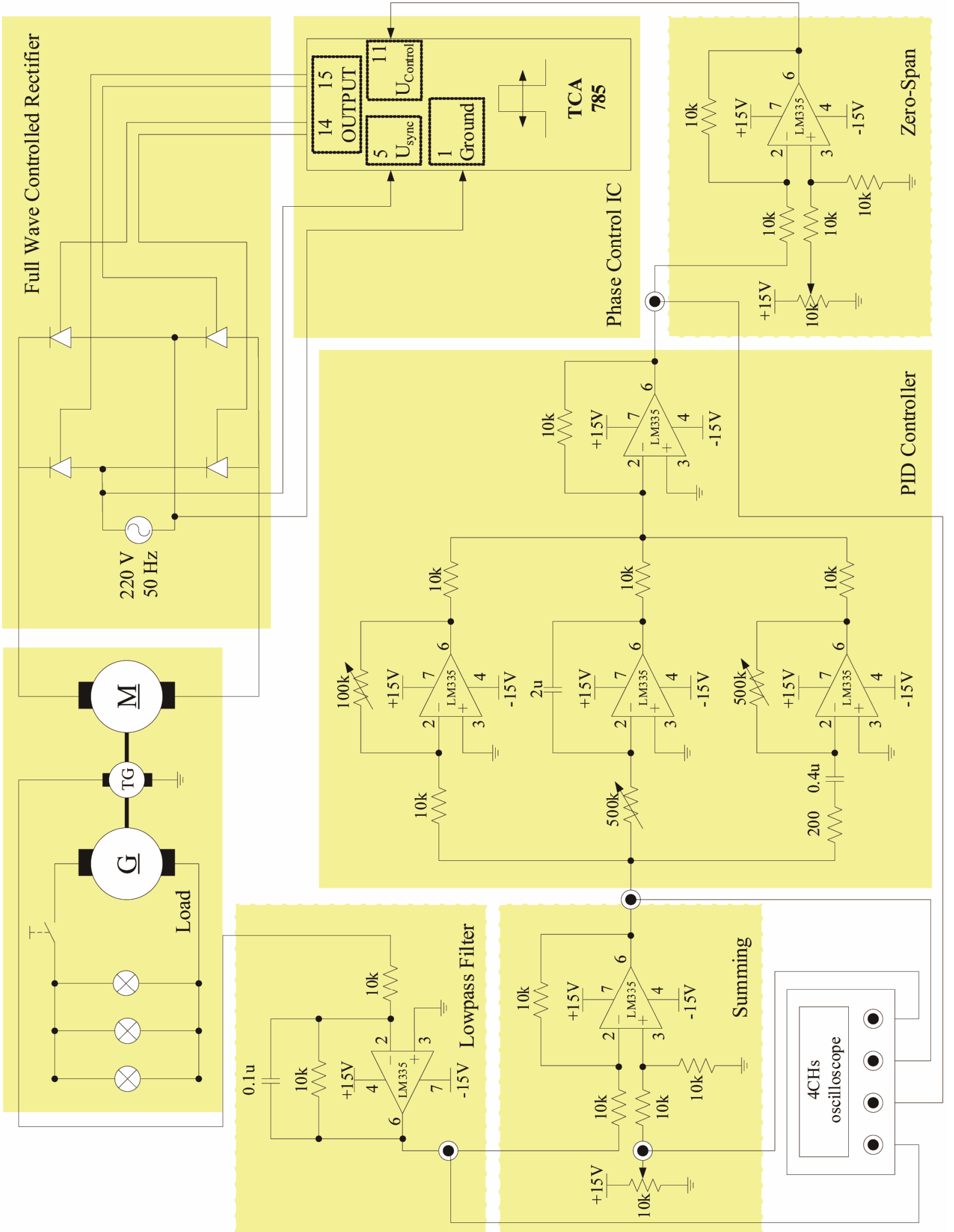

Figure 9. Circuit diagram representing the experimental setup. 


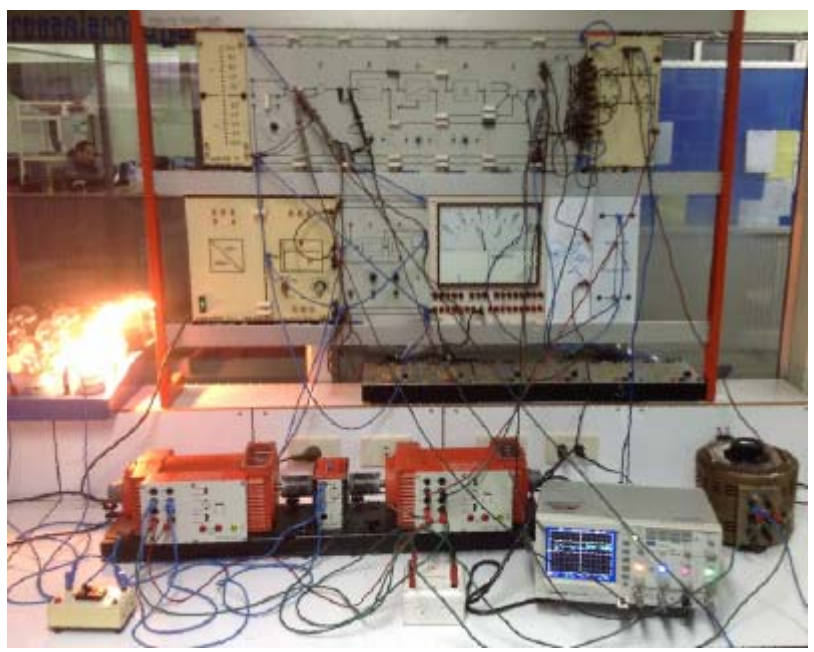

Figure 10. Experimental setup.

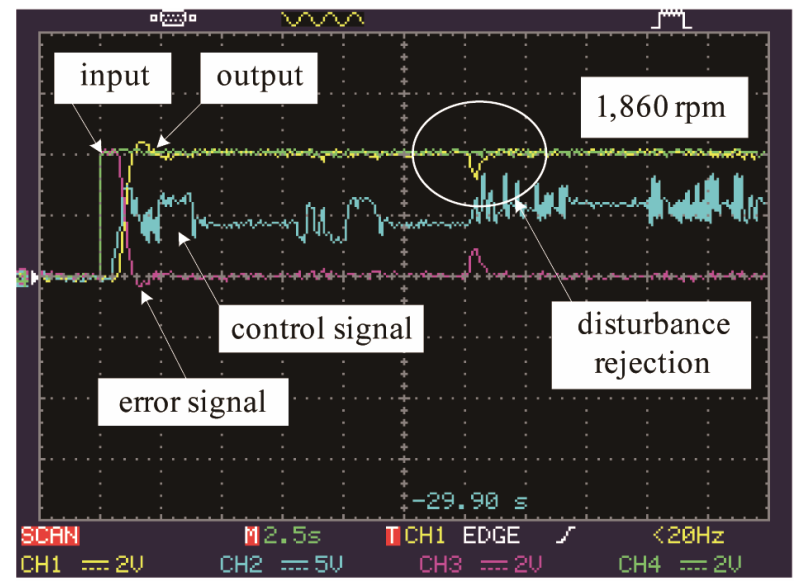

Figure 11. Experimental results.

tween the simulation results and the experiment ones can be observed from Figures 8 and 11.

\section{Conclusion}

The current search (CS) metaheuristics has been proposed in this paper. The CS algorithms have been conceptualized from the electric current flowing through electric networks for optimization problems. Algorithms of the CS have been elaborately described. Performance evaluation of the CS has been assessed by testing against eight well-known optimization problems. With two powerful strategies, i.e., exploration and exploitation, the proposed CS has provided superior search performances to GA, PSO, and ATS. The CS has been applied to design the optimum PID controller for the dc motor speed control system. An experimental bed of a closed loop dc motor speed control system was constructed at the laboratory. As results, the optimum PID controller can be obtained by the CS. Very good agreement between the simulation and experimental results has been also proposed.
This can be concluded that the optimum PID controller for the dc motor speed control system can be successfully obtained by the proposed CS metaheuristics. For the future trends of this work, the convergence proofs of the CS metaheuristics will be proposed. Moreover, the CS is still needed to be applied to various realworld engineering optimization among discrete, combinatorial, as well as multiobjective Pareto optimization problems especially in control engineering domain including model identification, system stabilization, and control synthesis.

\section{REFERENCES}

[1] F. Glover and G. A. Kochenberger, "Handbook of Metaheuristics," Kluwer Academic Publishers, Dordrecht, 2003.

[2] E. G. Talbi, "Metaheuristics Forn Design to Implementation," John Wiley \& Sons, Hoboken, 2009. doi: $10.1002 / 9780470496916$

[3] X. S. Yang, "Nature-Inspired Metaheuristic Algorithms," Luniver Press, 2010.

[4] D. E. Goldberg, "Genetic Algorithms in Search Optimization and Machine Learning," Addison Wesley Publishers, Boston, 1989.

[5] MathWorks, "Genetic Algorithm and Direct Search Toolbox: For Use with MATLAB," User's Guide, Version 1, MathWorks, Natick, Mass, 2005.

[6] M. Dorigo and T. Stützle, "Ant Colony Optimization," MIT Press, Cambridge, 2004. doi:10.1007/b99492

[7] J. Kennedy and R. Eberhart, "Particle Swarm Optimization," IEEE Proceedings of the International Conference on Neural Networks, Vol. 4, 1995, pp. 1942-1948. doi:10.1109/ICNN.1995.488968

[8] Z. W. Geem, "Recent Advance in Harmony Search Algorithm," Studies in Computational Intelligence, Springer, Berlin, 2010. doi:10.1007/978-3-642-04317-8

[9] X. S. Yang, "Firefly Algorithms for Multimodal Optimization, Stochastic Algorithms," Foundations and Applications SAGA 2009, Lecture Notes in Computer Sciences, Vol. 5792, 2009, pp. 169-178. doi:10.1007/978-3-642-04944-6 14

[10] R. Oftadeh, M. J. Mahjoob and M. Shariatpanahi, “A Novel Mata-Heuristic Optimization Algorithm Inspired by Group Hunting of Animals: Hunting Search," Computers and Mathematics with Applications, Vol. 60, No. 7, 2010, pp. 2087-2098. doi:10.1016/j.camwa.2010.07.049

[11] X. S. Yang and S. Deb, "Cuckoo Search via Lévy Flights," In: Proceedings of the World Congress on Nature and Biologically Inspired Computing, IEEE Publications, 2009, pp. 210-214.

[12] X. S. Yang and S. Deb, "Engineering Optimization by Cuckoo Search," International Journal of Mathematical Modeling and Numerical Optimization, Vol. 1, No. 4, 2010, pp. 330-343. doi:10.1504/IJMMNO.2010.035430

[13] X. S. Yang, "A New Metaheuristic Bat-Inspired Algorithm," In: J. R. Gonzalez, et al., Eds., Nature Inspired 
Cooperative Strategies for Optimization (NISCO 2010), Springer, Berlin, 2010, pp. 65-74.

[14] S. Kirkpatrick, C. D. Gelatt and M. P. Vecchi, "Optimization by Simulated Annealing," Science, Vol. 220, No. 4598, 1983, pp. 671-680. doi:10.1126/science.220.4598.671

[15] P. J. M. van Laarhoven and E. H. L. Aarts, "Simulated Annealing: Theory and Applications," Kluwer Academic Publishers, Dordrecht, 1987. doi:10.1007/978-94-015-7744-1

[16] F. Glover, "Tabu Search-Part I," ORSA Journal on Computing, Vol. 1, No. 3, 1989, pp. 190-206.

[17] F. Glover, "Tabu Search-Part II," ORSA Journal on Computing, Vol. 2, No. 1, 1990, pp. 4-32. doi:10.1287/ijoc.2.1.4

[18] S. Sujitjorn, T. Kulworawanichpong, D. Puangdownreong and K.-N. Areerak, "Adaptive Tabu Search and Applications in Engineering Design," Frontiers in Artificial Intelligent and Applications, IOS Press, Amsterdam, 2006.

[19] D. Puangdownreong, T. Kulworawanichpong and S. Sujitjorn, "Finite Convergence and Performance Evaluation of Adaptive Tabu Search," In: Lecture Notes in Artificial Intelligence, Springer-Verlag, Heidelberg, 2004, pp. 710-
717.

[20] A. Sukulin and D. Puangdownreong "A Novel MetaHeuristic Optimization Algorithm: Current Search," Proceedings of the 11th WSEAS International Conference on Artificial Intelligence, Knowledge Engineering and Data Bases (AIKED '12), Cambridge, 2012, pp. 125-130.

[21] D. Puangdownreong and A. Sukulin, "Obtaining an Optimum PID Controllers for Unstable Systems Using Current Search," International Journal of Systems Engineering, Applications \& Development, Vol. 2, No. 6, 2012, pp. 188-195.

[22] D. Puangdownreong, "Application of Current Search to Optimum PIDA Controller Design," Intelligent Control and Automation, Vol. 3, No. 4, 2012, pp. 303-312.

[23] M. M. Ali, C. Khompatraporn and Z. B. Zabinsky, “A Numerical Evaluation of Several Stochastic Algorithms on Selected Continuous Global Optimization Test Problems," Journal of Global Optimization, Vol. 31, No. 4, 2005, pp. 635-672. doi:10.1007/s10898-004-9972-2

[24] K. Ogata, "Modern Control Engineering," Prentice Hall, New Jersey, 2010.

[25] MathWorks, "System Identification Toolbox," User's Guide, Version 7.2, 2008. 